\title{
The Stabilizer Design for a Drone-Mounted Camera Gimbal System Using Intelligent-PID Controller and Tuned Mass Damper
}

\author{
Min $\mathrm{Kim}^{1}$, Gi-Sig Byun ${ }^{1}{ }^{1}$, Gwan-Hyung Kim ${ }^{2}$, Myoung-Hoon Choi ${ }^{1}$ \\ ${ }^{l}$ Dept. of Control and Instrumentation Engineering, Pukyong Univ., \\ Nam-gu, Busan, South Koera \\ ${ }^{2}$ Dept. of Computer Engineering, Tongmyong Univ., Yongdang-dong, Busan, \\ South Koera \\ kmmate@pukyong.ac.kr \\ "1gsbyun@pknu.ac.kr \{corresponding author\}
}

\begin{abstract}
Drones in flight make a variety of vibration frequencies. Therefore, gimbal system is needed to get clean images from drone-mounted cameras. The system consists of two things: one is a structure to support a camera module, and another is a stabilizer to block outside vibrations and keep the right angle. The purpose of this paper focuses on the system's dynamics model and the application of a traditional PID controller. Furthermore, the paper compares the traditional PID and intelligent-PID without any dynamics model through the simulation made of MATLAB/Simulink.
\end{abstract}

Keywords: drone, gimbal system, stabilizer, Intelligent-PID controller, tuned mass damper

\section{Introduction}

Such industries including drones, self-driving cars and ships recently have been emerging in a new engine for the future. Further drones have been developed and provided at a rapid pace beyond our expectations. It is also gaining grounding roles in disaster prevention, agriculture, management in industrial settings, sport broadcasting, and movie production - with its utility already having been proved.

A drone's gimbal system has the following equipment: one is a camera module and another is a stabilized drive system. Both of these remove camera vibrations and move camera equipment to direct positions in spite of the camera's vibration.

The system's stabilizer needs two controls in order to reduce vibrations that a drone makes to camera modules. One is rotary motion control in terms of the vibrations of rolling, pitching, and yawing. Another is linear motion control regarding the vibrations of surging, swaying, and heaving. PID control or Proportional-Integral-Derivative control is commonly used for motors that control rotary motion such as rolling, pitching, and yawing. However, the control's parameters should be adjusted if time delay occurs or system characteristics change. In this regard, Intelligent-PID control is applied in this paper. Also, in the case of residual vibration problems, this paper tries to set tuned mass damper into link structures composed of the Gimbal system and reduce the vibration of the linear motion.

\section{Gimbal System Model}

The Gimbal system is set in a camera to get clean images from a drone while the camera moves along its six axes such as rolling, pitching, yawing, surging, swaying, and 
heaving as shown below Figure 1. The system includes the structure built-in tuned mass damper at every axis of link, direct driving motor to control the rotary motion, Euler attitude angle measurement sensors, and DSP controller for computing algorithm. Usually, three-axis linear motion and three-axis rotary motion are symmetric. Therefore, this paper formulates a pattern model in terms of the axis of linear and rotary motion and designs stabilizing controller [1-3].

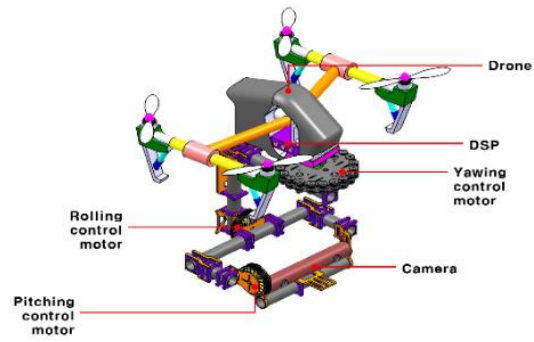

(a)

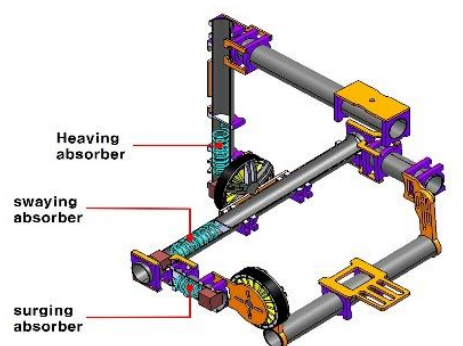

(b)

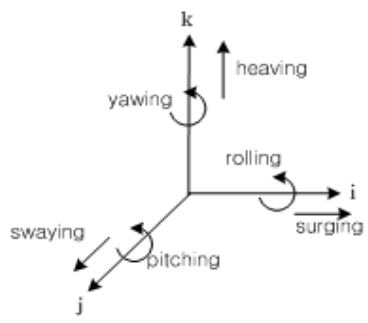

(c)

Figure 1. (a) Drone/Gimbal System (b) Gimbal Links with TMD (c) 6-Axis Motion of the Gimbal

First, for a derivation of the equation of motion, including Gimbal's rotary motion in terms of rolling, pitching and yawing, where a drone's coordinate system is $\mathrm{D}(i, j, k)$ and Gimbal's coordinate system is $\mathrm{G}(r, p, y)$, acceleration vector in terms of every coordinate system's axis is identified as the formula below.

$$
\overrightarrow{\omega_{D}}=\left[\begin{array}{l}
\omega_{i} \\
\omega_{j} \\
\omega_{k}
\end{array}\right], \quad \overrightarrow{\omega_{G}}=\left[\begin{array}{c}
\omega_{r} \\
\omega_{p} \\
\omega_{y}
\end{array}\right]
$$

After this, derivation of rotational-motion equation regarding drive motor in $p$-axis is followed as in Figure 2. When $p$-axis rotates as much as angle $\epsilon$, gimbal-axis' acceleration if we plot on gimbal-axis' acceleration as a function of drone-axis, it is as in the formula below.

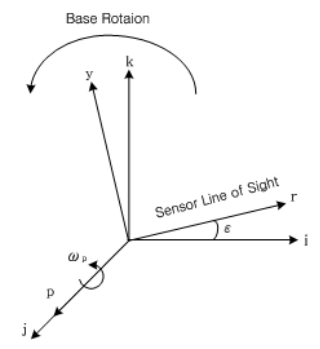

Figure 2. A Single-Axis Rotation Motion for Drone/Gimbal System

$$
\begin{array}{r}
\omega_{r}=\omega_{i} \cos \epsilon-\omega_{k} \sin \epsilon \\
\omega_{p}=\omega_{j}+\dot{\epsilon} \\
\omega_{y}=\omega_{i} \sin \epsilon+\omega_{k} \cos \epsilon
\end{array}
$$

Gimbal is as in the inertial matrix below.

$$
J=\left[\begin{array}{ccc}
J_{r} & J_{r p} & J_{r y} \\
J_{r p} & J_{p} & J_{y p} \\
J_{r y} & J_{y p} & J_{y}
\end{array}\right]
$$

Where $J_{r}, J_{p}, J_{y}$ is inertia moment about $r, p, y$-axis and $J_{r p}, J_{r y}, J_{y p}$ is deviations moment, according to the law of Newton, torque is as in the equation below. 


$$
\vec{T}=\frac{d}{d t}(\vec{P})+\overrightarrow{\omega_{G}} \times \vec{P}
$$

And then, $\vec{P}$ is quantify of motion and is defined as follows.

$$
\vec{P}=J \overrightarrow{\omega_{G}}=\left[\begin{array}{l}
J_{r} \omega_{r}+J_{r p} \omega_{p}+J_{r y} \omega_{y} \\
J_{r p} \omega_{r}+J_{p} \omega_{p}+J_{y p} \omega_{y} \\
J_{r y} \omega_{r}+J_{y p} \omega_{p}+J_{y} \omega_{y}
\end{array}\right]=\left[\begin{array}{c}
P_{r} \\
P_{p} \\
P_{y}
\end{array}\right]
$$

So, torque in terms of every axis of gimbal is followed as formula (4) through multiplying acceleration vector by momentum vector.

$$
\vec{T}=\left[\begin{array}{l}
\dot{P}_{r}+\omega_{p} P_{y}-\omega_{y} P_{p} \\
\dot{P_{p}}+\omega_{p} P_{r}-\omega_{y} P_{y} \\
\dot{P_{y}}+\omega_{p} P_{p}-\omega_{y} P_{r}
\end{array}\right]
$$

Further, torque equation of gimbal's p-axis is as follows.

$$
\tau=\dot{P_{p}}+\omega_{y} P_{r}-\omega_{r} P_{y}
$$

Among them, $\tau$ is torque linked from motor by substituting formula (5) and is defined as below.

$$
J_{p} \dot{\omega}_{p}=\tau+\left(J_{y}-J_{r}\right) \omega_{r} \omega_{y}+J_{r y}\left(\omega_{r}^{2}-\omega_{y}^{2}\right)-J_{y p}\left(\dot{\omega}_{y}-\omega_{p} \omega_{r}\right)-J_{r p}\left(\dot{\omega}_{r}-\omega_{p} \omega_{y}\right)
$$

If weight distribution in terms of gimbal-axis is asymmetric, deviationsmoment is 0 or $J_{r p}=J_{r y}=J_{y p}=0$. formula (8) is defined as below formula.

$$
J_{p} \dot{\omega}_{p}=\tau+\left(J_{y}-J_{r}\right) \omega_{r} \omega_{y}
$$

And, formula (2)'a $\omega_{r}, \omega_{y}$ is substituted and gimbal $p$-axis's rotational motion equation including drone's basic axis is as in the following formula.

$$
J_{p} \dot{\omega}_{p}=\tau+\left(J_{y}-J_{r}\right) \cdot\left(\omega_{i} \cos \epsilon-\omega_{k} \sin \epsilon\right) \cdot\left(\omega_{i} \sin \epsilon+\omega_{k} \cos \epsilon\right)
$$

If torque $\tau$ is assumed that it is generated by direct drive DC motor in formula (10), dynamic equation in terms of the motor's circuit is defined as below differential equation.

$$
\begin{aligned}
& e_{a}(t)=R_{a} i_{a}(t)+L_{a} \frac{d i_{a}}{d t}+e_{b}(t) \quad(11) \quad e_{b}(t)=K_{e} \omega(t) \\
& \tau(t)=K_{t} i_{a}(t) \\
& \tau(t)=J_{m} \frac{d \omega}{d t}+b_{m} \omega(t)+T_{D}(t)
\end{aligned}
$$

In the differential equation, $J_{m}=J_{a}+J_{L}$ is motor's inertia and link's inertia and $b_{m}=B+B_{L}$ is motor's coefficient of friction and gimbal link's coefficient of friction. However, in this paper, it is ignored.

The gimbal system including the rotary motion equation (10) about gimbal link derived by the above formula and driving equation (11)-(14), generating link's rotational torque is as in the below diagram. 


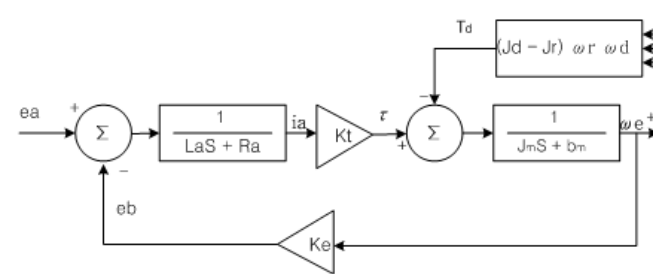

Figure 3. The Block Diagram of Rotation Motion Gimbal System

Also, a drone's electric motor makes vibrations of surging, swaying, and heaving. Therefore, it is regarded that a tuned mass damper is an effective device to block that vibration. TMD is a kind of vibration absorber and it blocks any vibration to the main device to make sure frequencies are constant and to protect it from external impacts by using additional absorption systems on the main device. The motion equation related with gimbal equipped with the turned mass damper is in as Figure 4 and formula (15) [4].

$$
\left[\begin{array}{cc}
m_{1} & 0 \\
0 & m_{2}
\end{array}\right]\left(\begin{array}{l}
\ddot{h_{1}} \\
\ddot{h_{2}}
\end{array}\right)+\left[\begin{array}{cc}
C_{1}+C_{2} & -C_{2} \\
-C_{2} & C_{2}
\end{array}\right]\left(\begin{array}{l}
\dot{h_{1}} \\
\dot{h_{2}}
\end{array}\right)+\left[\begin{array}{cc}
k_{1}+k_{2} & -k_{2} \\
-k_{2} & k_{2}
\end{array}\right]\left(\begin{array}{l}
h_{1} \\
h_{2}
\end{array}\right)=\left(\begin{array}{c}
F_{1} \\
0
\end{array}\right) \sin \omega t
$$

$m_{1}, c_{1}, k_{1}$ is mass, damping factor and rigidity in terms of gimbal-axis and $m_{2}, c_{2}, k_{2}$ is mass, damping factor and rigidity regarding TMD. $h_{1}$ is the displacement of gimbal, $h_{2}$ is the displacement of TMD and $f$ is vibration shaking gimbal. If the harmonic function of this formula is assumed, steady state solution is as in the formula below.

$$
\begin{aligned}
& h_{j}(t)=H_{j} e^{i \omega t}, \quad j=1,2 \\
& {\left[\begin{array}{cc}
k_{1}+k_{2}-m_{1} \omega^{2}+i \omega\left(c_{1}+c_{2}\right) & -k_{2}-i \omega c_{2} \\
-k_{2}+i \omega c_{2} & k_{2}-m_{2} \omega^{2}+i \omega c_{2}
\end{array}\right]\left(\begin{array}{c}
H_{1} \\
H_{2}
\end{array}\right)=\left(\begin{array}{c}
F_{1} \\
0
\end{array}\right)}
\end{aligned}
$$

Also, linear vibration model of gimbal-axis absorbing vibrations by using TMD is as in the formula below.

$$
H_{1}=\frac{k_{2}-m_{2} \omega^{2}+i \omega c_{2}}{\left(k_{1}+k_{2}-m_{1} \omega^{2}+i \omega c_{1}+i \omega c_{2}\right)\left(k_{2}-m_{2} \omega^{2}+i \omega c_{2}\right)-\left(k_{2}+i \omega c_{2}\right)^{2}} F_{1}
$$

\section{Intelligent-PID Controller Design}

This paper describes how to design an intelligent-PID controller without any modelling in order to design a controller for rotary motion gimbal link [5-7].

Usually, system dynamic equation is described as the function of input $u(t)$ and system output $y(t)$ as the formula below.

$$
y^{(m)}(t)=g\left(y^{(m-1)}(t), \ldots y(\dot{t}), t(t), u^{(l)}(t), \ldots, u(\dot{t}), u(t)\right)
$$

This formula below is very suitable to be measured for a short time.

$$
y^{(n)}(t)=G(t)+\alpha u(t)
$$

In the above formula, $\mathrm{n}$ is usually 1 or $2 . \alpha$ is the value that a designer puts. $G(t)$ is $y(t), u(t)$ and higher order differential function and it is an unknown term including all of information from the controlled system. Also, it is measured in real time by using input $u(t)$ and measured value $y^{(n)}(t)$.

If $G(t)$ can be assumed, in a closed-loop control system, control input set is decided as the below formula (20) in order for exact output. Further, in the case of $n=2[2 \sim 4]$.

$$
u(t)=\frac{1}{\alpha}\left(-G(t)+\ddot{y}^{*}(t)+K_{P} e(t)+K_{I} \int e(\tau) d \tau+K_{D} \dot{e}(t)\right.
$$


In the above formula

$\ddot{y}^{*}(t)$ is a signal that the output of the controlled system should follow.

$e(t)=y^{*}-y(t)$ is follow-up error

$K_{P}, K_{I}, K_{D}$ is the general gains of PID

Also, $G(t)$ is $y^{(n)}(t)-\alpha u(t)$ from the above formula (20) and closed-loop response's quintic equation is found by the substitution of the formula (21).

$$
\ddot{e}(t)+K_{P} e(t)+K_{I} \int e(\tau) d \tau+K_{D} \dot{e}(t)=0
$$

With substitution on both sides of that, closed-loop characteristic equation follows as the formula below (23).

$$
e(s)=s^{3}+K_{D} s^{2}+K_{P} s+K_{I}
$$

So, if $K_{P}, K_{I}, K_{D}$ is set for formula (23), it becomes $e(t) \rightarrow 0(t \rightarrow \infty)$.

In fact, the formula (21)'s $G(t)$ is unknown and it is necessary to compute it in realtime. If $u(t-h)$ is used as much as $h(>0)$ instead of formula (20)'s $u(t), G(t)$ is assumed as in the below formula (24).

$$
\left.G(t)\right|_{e}=\ddot{y}(t)-\alpha u(t-h), \quad(u(t-h) \approx u(t))
$$

Actual inhibit input is set as in the below formula (25) for the exact output of the controlled system by substituting this value in the formula (21).

$$
u(t)=\frac{1}{\alpha}\left(-\ddot{y}(t)+\alpha u(t-h)+\ddot{y}^{*}(t)+K_{P} e(t)+K_{I} \int e(\tau) d \tau+K_{D} \dot{e}(t)\right)
$$

Figure 5 indicates the controlling system structure implemented by this controller.

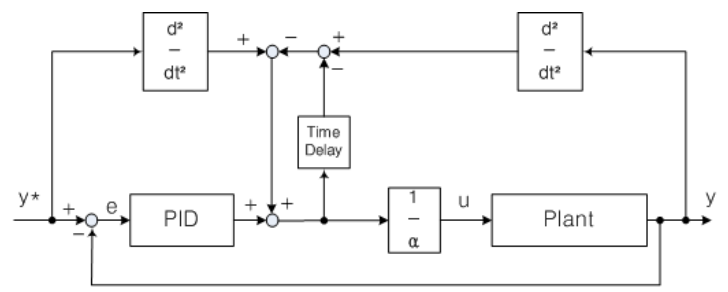

Figure 5. Intelligent-PID Control System

\section{Simulation and Results}

Chapter 4 compares the outcomes of a PID controller applied on a modeled plant in chapter 2, which is used for stabilizing the vibrations of gimbal's rolling, pitching, and yawing and the stabilization performance of Intelligent PID suggested in chapter 3, depending on plant situation can be changed as circumstances change. DC motor parameters are set in as Table 1 below for simulating how controllers work.

Table 1. Parameters and Variables of DC Motor

\begin{tabular}{|c|c|}
\hline Item & Value \\
\hline Resistance of the motor & $R_{a}=4.5[\Omega]$ \\
\hline Inductance of the motor & $L_{a}=0.003[\mathrm{H}]$ \\
\hline Motor's moment of inertia & $J_{m}=0.0017\left[\mathrm{Kgm}^{2}\right]$ \\
\hline Motor electrical constant & $K_{e}=0.85[\mathrm{~V} / \mathrm{rad} / \mathrm{sec}]$ \\
\hline Motor mechanical constant & $K_{T M}=0.85[\mathrm{Nm} / \mathrm{A}]$ \\
\hline
\end{tabular}


If the gimbal system equipped camera's weight is $1[\mathrm{Kg}]$ and it is a body of revolution of $14[\mathrm{~cm}]$, inertia moment is $J_{L}=\frac{1}{2} \mathrm{Mr}^{2} \equiv 9.8 \times 10^{-3}\left[\mathrm{Kgm}^{2}\right]$. Therefore, the system's inertia moment is $J_{m}^{*}=J_{m}+J_{L}=11.5 \times 10^{-3}\left[\mathrm{Kgm}^{2}\right]$ added by the motor's inertia moment. This simulation excludes coefficient of friction.

First, the traditional PID controller multiplies error signal $(e(t))$ or plant's output signal $(y(t))$ by proportion $\left(K_{P}\right)$, integral calculus $\left(K_{I}\right)$, differential $\left(K_{D}\right)$ and constant and has control signal $u(t)$ made of that algorithm as in Figure 6(a) below. Among them, the controller's gain values estimate $K_{P}=0.96, K_{I}=10.7, K_{D}=0.0001$ by Zigler-Nichols method. When target input angular speed $\left(y^{*}(t)=\omega_{c}\right)$ is $0.35[\mathrm{rad} / \mathrm{sec}]$, the drone body's $i$-axis angular speed changed into $\omega_{i}=0.09[\mathrm{rad} / \mathrm{sec}]$, its $k$-axis angular speed turned into $\omega_{k}=0.17[\mathrm{rad} / \mathrm{sec}]$, its $j$-axis angular speed transformed into $\omega_{j}=$ $0 \sim 0.61[\mathrm{rad} / \mathrm{sec}]$ and gimbal $p$-axis's angular speed output $\left(y(t)=\omega_{p}\right)$ in terms of change of disturbance are simulated as in Figure 6(b) below.

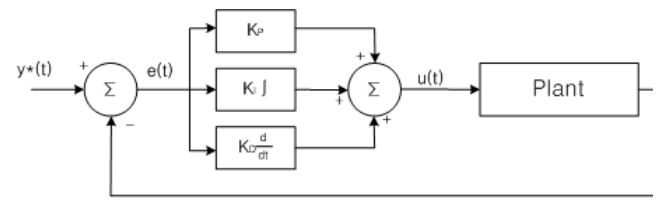

(a)

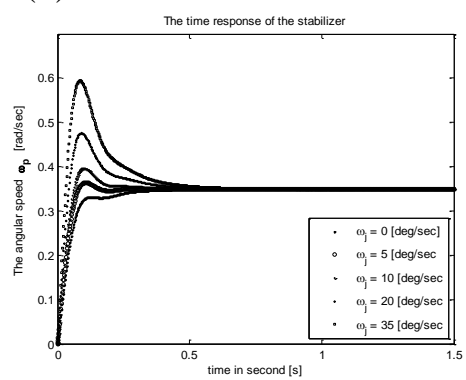

(b)

\section{Figure 6. (a) PID Control System, (b) PID Control System Response}

First, a controller is designed while it uses quintic equation (23)'s stabilization condition to solve $K_{P}=12, K_{I}=8, K_{D}=6$ and time delay $h$ is put in 0.01 and $\alpha$ is 1 or $\alpha=1$, according to the design of Intelligent-PID that this paper suggests. Then, that is made of controlling system as in Figure 5. The experimental condition that this paper has already suggested is substituted and the outcomes of the simulation gain the response characteristics as Figure 7 here.

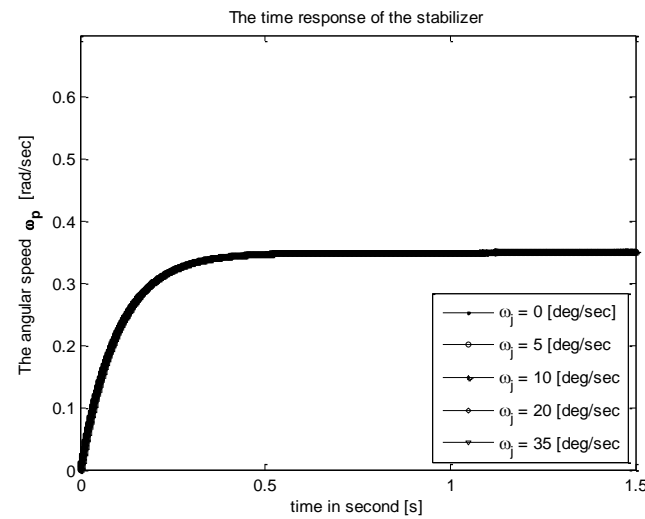

Figure 7. Intelligent-PID Control System Response

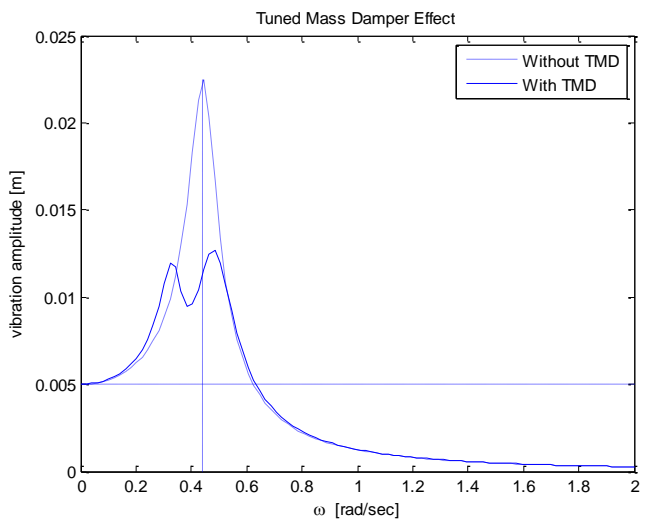

Figure 8. Gimbal Vibration Response with TMD

With the outcomes, a traditional PID controller expects that a drone's angular speed response characteristics in terms of change of disturbance have worsened. However, Intelligent PID has highly strengthened response characteristics to the circumstances 
change. Furthermore, in the case of the design of the intelligent PID, it can select controller parameters without any dynamic model. It is also great in control performance. Also, a tuned mass damper is designed to absorb the vibrations of surging, swaying, and heaving, which gimbal's motor makes and its parameter is followed as the Figure below. First, TMD's natural frequency and damping rate is in as follows [8].

$$
\omega_{2}=\sqrt{\frac{k_{2}}{m_{2}}}
$$

$$
\zeta_{2}=\frac{c_{2}}{2 m_{2} \omega_{2}}
$$

Natural frequency ration $\gamma-$ which is TMD and gimbal's natural frequency - and mass ration $u$ - which is TMD and gimbal's mass - are followed as the formula below (28) and (29).

$$
\gamma=\frac{\omega_{2}}{\omega_{1}}
$$

$$
u=\frac{m_{2}}{m_{1}}
$$

Once mass ratio is decided, TMD's optimum frequency ratio and damping ratio in terms of the gimbal affected by irregular vibrations is in as follows.

$$
\gamma_{\text {opt }}=\frac{1}{1+u} \quad \text { (30) } \quad \epsilon_{\text {opt }}=\frac{1}{2} \sqrt{\frac{1.5 u}{1+3 u}}
$$

Optimum frequency ratio in terms of the mass ratio $1.5 \%$ of TMD and TMD's mass, damping factor and rigidity regarding optimum damping rate are designed and with these measures, response characteristics about the linear vibrations of gimbal's swaying, surging, and heaving are formulated as shown in Figure 8.

The natural frequency of gimbal is changed and its amplitude of vibration decreases effectively as TMD is set in every axis' link of gimbal through the above outcomes.

\section{Conclusion}

Recently, drones have been gaining grounding roles in disaster prevention, agriculture, management in industrial settings, sport broadcasting, and movie production, with its utility already having been proven in these fields. Furthermore, the drone gimbal system is a device that can move to follow its target, support camera equipment, and block any vibrations to the camera, even though the camera equipment are affected by these vibrations. Also, the PID controlling method is usually used to control rotational vibration while a stabilizer controls the vibrations of the gimbal system. However, the readjustment of its parameters should be needed if time is delayed to the controlled system or characteristics such as disturbance are transformed. Therefore, this paper aims to overcome these problems while applying intelligent-PID controlling methods on the gimbal system's drive system for a stabilizer. Furthermore, vibration control is necessary to protect the gimbal system from linear vibrations. In this background, this paper focuses on setting the tuned mass damper in the gimbal system's link, considering it is a very effective manual vibration controller.

In this regard, this paper identifies that an Intelligent-PID controlling method has very strong Servo characteristics in terms of rotational vibrations of the gimbal system, even as external circumstances are changed. It finds the effectiveness of TMD as it improves the ability of absorption of vibrations regarding linear vibrations. Therefore, an experiment must be scheduled to get clean images as applying these research results on a drone gimbal system stabilizer.

"This work was supported by a Research Grant of Pukyong National University (2015 year)" 


\section{References}

[1] B. Ekstrand, "Equations of motion for a two-axes gimbal system," Aerospace and Electronic System, IEEE Transactions on, vol. 37, no. 3, (2001), pp.1083-1091.

[2] M. Abdo, A. R. Toloei, A. R. Vali, M. R. Arvan, "Modeling, control and simulation of cascade servo system for one axis gimbal mechanism," International Journal of Engineering, vol. 27, no. 1, (2014), pp.157-170.

[3] L. Esfandiari, "Modeling and Analysis of Dynamic Systems, Second Edition, CRC Press, (2014).

[4] W. Y. Cha, C. K. Chun, S. G. Park and H. H. Han, "Developing tuned mass damper of adjustable damping type to control the vibration of medical robots," Trans. Korean Soc. Noise Vib. Eng., no 24, vol. 9, (2014), pp. 706-715.

[5] M. Fliess and C. Join, "Intelligent PID controllers," in Proc. of the 16th Mediterranean Conference on Control and Automation, (2008), pp. 326-331.

[6] M. Fliess and C. Join, "Model-free control and intelligent PID controllers: toward a possible trivialization of nonlinear," in Proc. of the 15th IFAC Symposium on System Identification, (2009), pp. 1531-1541.

[7] B. D. Novel, M. Fliess, C. Join, H. Mounier and B. Steux, "A mathematical explanation via" intelligent" PID controllers of the strange ubiquity of PIDs," in 18th Mediterranean Conference on Control and Automation, Marrakech, Morocco, (2010).

[8] R. Rana and T. T. Soong, "Parametric study and simplified design of tuned mas dampers", Engineering Structures, vol. 30, no. 3, (1998), pp. 193-204.

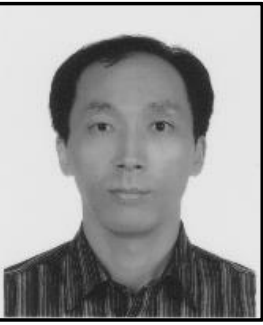

Min Kim, he received his M.S. degree in Control \& Instrumentation Engineering from Pukyong National University in 2012. He also has a Doctoral degree with the Department of Control \& Instrumentation Engineering, Pukyong National University. His research interests are signal processing, Embedded System Design, Sensor Network.

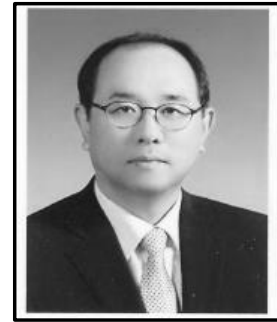

Gi-Sig Byun received his Ph.D. degree in Electrical Engineering from Wichita State University in 1990. Since 1990, he has been a professor with the Department of Control \& Instrumentation Engineering, Pukyong National University. His research interests are signal processing, Embedded System Design, Sensor Network, Digital Control.

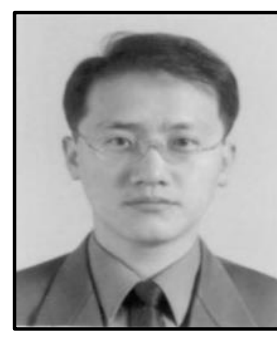

Gwan-Hyung Kim received his Ph.D. degree in Electrical Communication from Korea Maritime University in 2001. He is currently a Full-Time Instructor in Dept. of Computer Engineering, TongMyong University. His research interests are intelligent control, signal processing, and robotics.

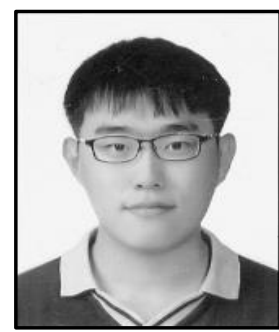

Myoung-Hoon Choi received his B.S. degree in Control \& Instrumentation Engineering from Pukyong National University in 2015. He also has a Master's degree with the Department of Control \& Instrumentation Engineering, Pukyong University, in 2015. He research interests includes Controller System, Stereoscopic Image Processing, Sensor Network, programming. 